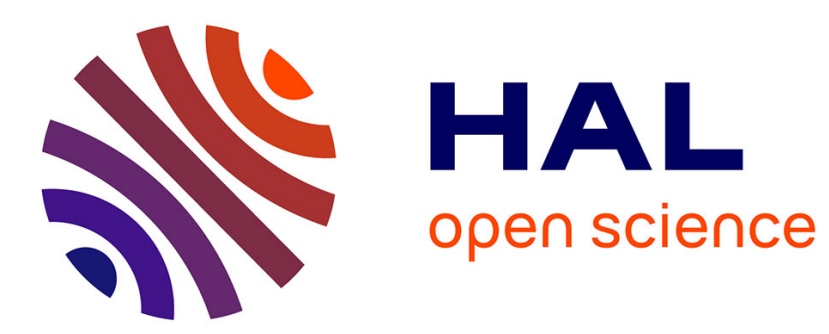

\title{
Echangeurs à caloducs pour la récupération de chaleur dans les processus industriels
}

S. Chaudourne

\section{To cite this version:}

S. Chaudourne. Echangeurs à caloducs pour la récupération de chaleur dans les processus industriels. Revue de Physique Appliquée, 1982, 17 (9), pp.625-632. 10.1051/rphysap:01982001709062500 . jpa00245040

\section{HAL Id: jpa-00245040 https://hal.science/jpa-00245040}

Submitted on 1 Jan 1982

HAL is a multi-disciplinary open access archive for the deposit and dissemination of scientific research documents, whether they are published or not. The documents may come from teaching and research institutions in France or abroad, or from public or private research centers.
L'archive ouverte pluridisciplinaire HAL, est destinée au dépôt et à la diffusion de documents scientifiques de niveau recherche, publiés ou non, émanant des établissements d'enseignement et de recherche français ou étrangers, des laboratoires publics ou privés. 


\title{
Echangeurs à caloducs pour la récupération de chaleur dans les processus industriels
}

\author{
S. Chaudourne \\ C.E.A., Service des Transferts Thermiques, 85X, 38041 Grenoble cedex, France
}

(Reçu le 21 septembre 1981, révisé le 15 janvier 1982, accepté le 19 avril 1982)

\begin{abstract}
Résumé. - On présente le principe et les avantages des échangeurs à caloducs pour la récupération de chaleur sur les fluides chauds rejetés, notamment dans les processus industriels.

Une méthode de calcul est présentée et les paramètres particuliers à l'utilisation des caloducs sont analysés.
\end{abstract}

Abstract. - Principle and advantages of heat pipe heat exchangers for heat recovery in industrial processes are presented.

A method of design is given and special features of these heat exchangers are studied.

\section{Notations.}

$A \quad$ Aire de la surface d'échange.

$C \quad$ Débit de capacité d'un fluide.

$C^{*} \quad$ Rapport $C_{\min } / C_{\max }$ des débits de capacité des deux fluides.

$E \quad$ Efficacité d'un échangeur.

$N \quad$ Nombre de caloducs dans l'échangeur.

NUT Nombre d'unités de transfert.

$\Delta P \quad$ Diminution de pression.

$Q \quad$ Flux de chaleur échangé.

$R \quad$ Nombre de rangées de caloducs dans l'échangeur.

$T \quad$ Température

$U$ Coefficient d'échange global.

\section{Indices}

c Côté chaud de l'échangeur.

e Entrée de l'échangeur.

f Côté froid de l'échangeur.

$i \quad i$-ième rangée de caloducs.

$\ell \quad$ Fluide intermédiaire.

$\max$ Maximum.

min Minimum.

s Sortie de l'échangeur.

t Tube caloduc.

1. Introduction. - 1.1 GÉNÉRALITÉs. - Les caloducs sont des dispositifs capables de transmettre des flux de chaleur importants avec de faibles écarts de températures. Un caloduc se présente sous la forme d'un tube revêtu intérieurement d'un matériau poreux, fermé hermétiquement et contenant un fluide en équilibre liquide-vapeur (Fig. 1). Le mécanisme cyclique évaporation-transfert de vapeur-condensation-retour du condensat à l'évaporateur permet d'atteindre des densités de flux axial transféré entre les extrémités du tube de plusieurs dizaines à plusieurs milliers de watts par $\mathrm{cm}^{2}$ de section [1], [2]. Le matériau poreux assure le retour du condensat, même en l'absence de gravité.

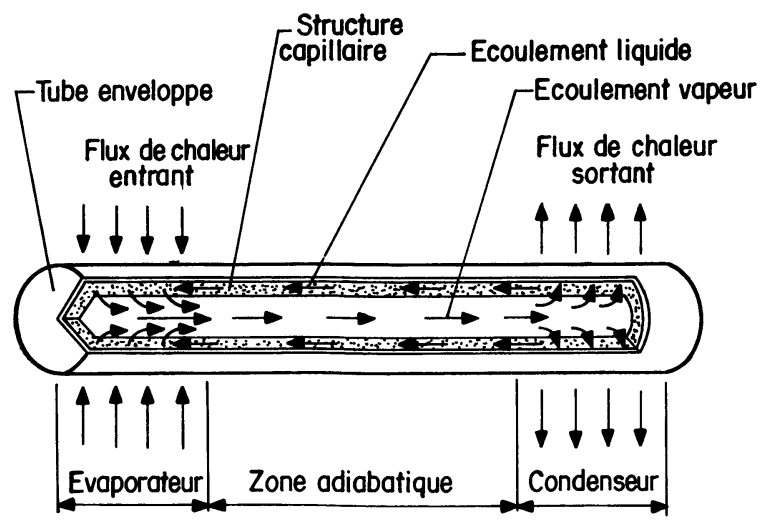

Fig. 1. - Principe du caloduc.

[Principle of a heat pipe.]

Les propriétés des caloducs sont utilisées dans certains échangeurs de chaleur pour transférer la chaleur récupérée sur un fluide chaud vers un fluide froid que l'on désire réchauffer avant utilisation. 
Le récupérateur se présente sous la forme d'une batterie de caloducs dont les évaporateurs plongent dans le courant de fluide chaud et les condenseurs dans le courant de fluide froid. Afin d'obtenir une surface d'échange suffisante, lorsque les fluides sont gazeux les tubes caloducs doivent être munis d'ailettes (Fig. 2), [3], [4].

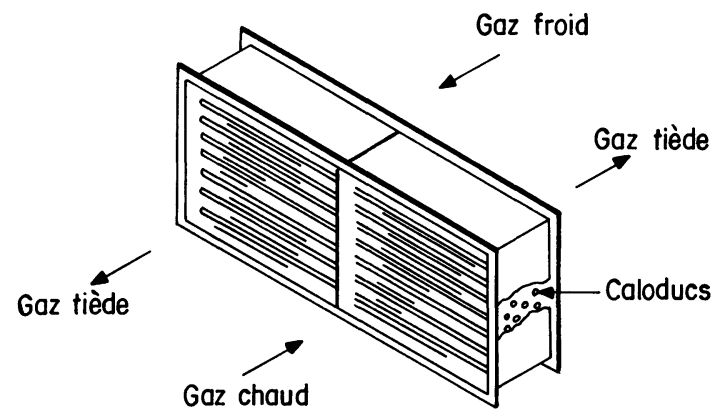

Fig. 2. - Schéma d'un échangeur à caloduc.

[Schematic of a heat pipe heat exchanger.]

Le choix du fluide placé dans les caloducs est fonction de la température de travail ; en effet, la vapeur étant toujours saturante, la température détermine la pression dans le caloduc. La température minimum correspond à la pression la plus faible en deçà de laquelle la vapeur est trop peu dense pour acheminer correctement la puissance demandée (blocage sonique). La température maximum est donnée par la pression maximum compatible avec la tenue mécanique de l'enveloppe, du queusot et des bouchons.

On peut ainsi dresser le tableau des domaines de

Tableau I. - Domaine de température des principaux fluides utilisés dans les caloducs.

[Temperature range for some heat pipe fluids.]

\begin{tabular}{|c|cc|}
\hline \multirow{2}{*}{ Fluide } & \multicolumn{2}{|c|}{$\begin{array}{c}\text { Domaine de } \\
\text { Température }\left({ }^{\circ} \mathrm{C}\right)\end{array}$} \\
\hline Ammoniaque & -60 & 60 \\
\hline Fréon 11 & -40 & 120 \\
\hline Acétone & -10 & 130 \\
\hline Ethano1 & -10 & 130 \\
\hline Methano1 & -30 & 130 \\
\hline Eau & 30 & 220 \\
\hline $\begin{array}{c}\text { Dowtherm A } \\
\text { Gilotherm D0 }\end{array}$ & 130 & 370 \\
\hline Mercure & 200 & 600 \\
\hline Soufre & 250 & 650 \\
\hline Potassium & 450 & 850 \\
\hline Sodium & 570 & 1000 \\
\hline
\end{tabular}

températures pour les fluides les plus courants (Tableau I).
Le choix des matériaux des tubes caloducs est déterminé par les problèmes de corrosion et de tenue mécanique. La corrosion doit être examinée du côté intérieur avec le fluide caloduc et du côté extérieur avec les fluides traversant l'échangeur. Ce dernier cas dépend de la composition et de la température des fluides et il doit faire l'objet d'une étude particulière à chaque réalisation.

Du côté intérieur, aucune corrosion par le fluide caloduc ne peut être tolérée car celle-ci est susceptible de dégager des gaz incondensables bloquant le fonctionnement du caloduc, [5].

Les matériaux usuels compatibles avec les fluides cités dans le tableau I sont indiqués dans le tableau II.

Tableau II. - Compatibilité des fluides utilisés avec le tube enveloppe des caloducs.

[Compatibility of fluids with container.]

\begin{tabular}{|c|c|}
\hline Fluide caloduc & $\begin{array}{l}\text { Matériau d'enveloppe usuel } \\
\text { compatible }\end{array}$ \\
\hline Ammoniaque & Aluminium - Acier inox \\
\hline Fréon 11 & Aluminium \\
\hline Acétone & Cuivre \\
\hline Methanol & Cuivre \\
\hline Ethanol & Cuivre \\
\hline Eau & Cuivre \\
\hline $\begin{array}{c}\text { Dowtherm A } \\
\text { Gilotherm DO }\end{array}$ & Acier inox - Cuivre \\
\hline Mercure & Acier inox $\left(T<500^{\circ} \mathrm{C}\right)-$ acier non allié \\
\hline Soufre & Acier inox AISI 316 (avec réserves) \\
\hline Potassium & Acier Inox - Nickel \\
\hline Sodium & Acier Inox \\
\hline
\end{tabular}

Les exigences parfois contradictoires entre la corrosion interne et la corrosion externe peuvent être satisfaites à l'aide de tubes bimétalliques.

Les échangeurs récupérateurs à caloducs sont actuellement fabriqués industriellement dans le domaine des températures ambiantes pour utilisation dans les installations de climatisation; le couple Aluminium-Fréon (Forane 11 en général) est le plus couramment utilisé.

Dans le domaine des températures moyennes $\left(150\right.$ à $\left.500^{\circ} \mathrm{C}\right)$ rencontrées en milieu industriel, les études de développement sont en cours et l'on s'oriente vers des caloducs en acier non allié ou en acier inoxydable avec fluide organique (type Gilotherm DO) ou mercure.

Enfin, dans le domaine des hautes températures $\left(600\right.$ à $\left.1200^{\circ} \mathrm{C}\right)$ rencontrées notamment dans l'industrie sidérurgique la recherche s'oriente vers des enveloppes mixtes métal-céramique et des fluides métalliques alcalins tels que potassium, sodium, lithium [6].

1.2 COMPARAISON AVEC LES ÉCHANGEURS RÉCUPÉRATEURS ClassiQues [7]. — CeuX-ci comprennent notamment :

- Les régénérateurs tournants constitués par une 
matrice poreuse, emmagasinant la chaleur qui se trouve alternativement en contact avec les gaz chauds et avec les gaz froids à réchauffer.

Ils présentent l'inconvénient par rapport aux récupérateurs à caloducs de nécessiter un apport d'énergie extérieur et de présenter une étanchéité imparfaite entre les deux circuits de gaz.

- Les batteries à fluide intermédiaire présentent une certaine analogie avec les caloducs dont le fluide interne joue le rôle de fluide intermédiaire.

Cependant elles ont également l'inconvénient d'utiliser une source d'énergie extérieure (pour la pompe de circulation du fluide intermédiaire). Elles ont aussi un moins bon coefficient de transfert que les échangeurs à caloducs car le fluide intermédiaire travaille en monophasique et non en diphasique comme dans les caloducs.

D’une manière générale, les avantages des récupérateurs à caloducs sont les suivants :

- Absence d'organe en mouvement.

- Durée de vie élevée.

- Aucun apport d'énergie extérieure.

- Aucun mélange des gaz chauds et froids.

- Souplesse dans la conception pour le dimensionnement comme pour les performances recherchées.

- Compacité.

- Réversibilité ou irréversibilité du sens de conduction selon la conception des caloducs (voir $\S 3.3$ ).

2. Méthode de calcul d'un échangeur à caloducs [8], [9]. - 2.1 GÉNÉRALITÉS. - Le calcul d'un échangeur à caloducs relève essentiellement des méthodes générales de calcul des échangeurs. L'aspect spécifique des caloducs intervient à deux niveaux :

1) Dans le calcul de la résistance thermique globale de l'échangeur où s'intègre la résistance du caloduc qui dépend de sa structure et des conditions dans lesquelles il travaille.

2) Dans le fait que la résistance thermique d'un caloduc ne peut être considérée comme approximativement constante et faible, qu'en deçà d'un certain flux limite au-delà duquel le caloduc se "bloque". Sa résistance devient alors brutalement très grande; il faudra donc s'assurer par le calcul que le flux limite ne risque pas d'être dépassé dans les conditions de fonctionnement. Ceci s'applique particulièrement à la phase de démarrage.

2.2 Principe du CAlcul. - La méthode que nous proposons consiste à considérer chaque rangée de caloducs de l'échangeur comme un ensemble de deux échangeurs couplés par le fluide caloduc : un évaporateur réchauffé par le fluide externe chaud et un condenseur refroidi par le fluide externe froid.

L'ensemble des rangées constituera une suite d'échangeurs associés en contre-courant global.

On utilisera pour calculer chaque échangeur élé- mentaire la méthode du Nombre d'Unités de Transfert (N.U.T.).

On fera ensuite usage des formules permettant de calculer l'efficacité globale de certaines associations d'échangeurs.

2.3 RAPPEL DE LA MÉTHODE N.U.T. - Le but de la méthode est de calculer l'efficacité $E$ de l'échangeur qui est le rapport du flux thermique qui le traverse au flux qui traverserait un échangeur à contre-courant de surface infinie, avec les mêmes conditions d'entrée des fluides.

Le flux réellement échangé a pour expression :

$$
Q=C_{\mathrm{c}}\left(T_{\mathrm{ce}}-T_{\mathrm{cs}}\right)=C_{\mathrm{f}}\left(T_{\mathrm{fs}}-T_{\mathrm{fe}}\right)
$$

dans laquelle les indices $c$ et $f$ se rapportent aux fluides chaud et froid respectivement, les indices e et $\mathrm{s}$ à l'entrée et la sortie de ces fluides.

$C$ est un débit de capacité : produit du débit masse du fluide par sa chaleur massique, $T$ est la température.

Lorsque la surface d'échange tend vers l'infini dans un échangeur à contre-courant, la température de sortie du fluide qui a le plus faible débit de capacité $\left(C_{\min }\right)$ tend vers la température d'entrée de l'autre fluide.

Le flux échangé à la limite, $Q_{\infty}$ s'écrit donc :

$$
\begin{array}{llll}
Q_{\infty}=C_{\mathrm{c}}\left(T_{\mathrm{ce}}-T_{\mathrm{fe}}\right) & \text { si } & C_{\mathrm{c}}=C_{\mathrm{min}} \\
Q_{\infty}=C_{\mathrm{f}}\left(T_{\mathrm{ce}}-T_{\mathrm{fe}}\right) & \text { si } & C_{\mathrm{f}}=C_{\min } .
\end{array}
$$

L'efficacité définie par $E=Q / Q_{\infty}$ s'écrit alors :

$$
\begin{aligned}
& E=\frac{T_{\mathrm{ce}}-T_{\mathrm{cs}}}{T_{\mathrm{ce}}-T_{\mathrm{fe}}} \text { si } C_{\mathrm{min}}=C_{\mathrm{c}} \\
& E=\frac{T_{\mathrm{fs}}-T_{\mathrm{fe}}}{T_{\mathrm{ce}}-T_{\mathrm{fe}}} \text { si } C_{\mathrm{min}}=C_{\mathrm{f}}
\end{aligned}
$$

et l'on a :

$$
Q=E \cdot C_{\mathrm{min}}\left(T_{\mathrm{ce}}-T_{\mathrm{fe}}\right) .
$$

Dans la méthode N.U.T., l'efficacité est calculée par des relations analytiques ou expérimentales en fonction du nombre d'unités de transfert de l'échangeur que l'on définit par la relation :

$$
N U T=\frac{U A}{C_{\min }}
$$

dans laquelle $U$ est le coefficient d'échange global rapporté à la surface d'échange $A$.

$E$ étant connu, on en déduit $Q$ par la relation (5) et $T_{\mathrm{cs}}, T_{\mathrm{fs}}$ par la relation (1).

Dans le cas où l'échangeur à calculer est un évaporateur ou un condenseur, la température du fluide diphasique, supposé pur, est constante; ceci, compte tenu de la relation (1) conduit à lui attribuer un débit de capacité infini.

Il en résulte que $C_{\min }$ est toujours le débit de capacité du fluide monophasique et que par ailleurs la 
relation entre $N U T$ et $E$ peut se mettre sous la forme analytique simple suivante [11] :

$$
E=1-\mathrm{e}^{-N U T}
$$

\subsection{Calcul De L'efFicaCité Globale De L'ÉCHAN-} GEUR. - 2.4.1 Expression générale. - Nous allons d'abord établir l'expression générale de l'efficacité globale $E$ de deux échangeurs couplés par un circuit fluide commun (Fig. 3). L'indice $\ell$ est relatif à ce fluide,

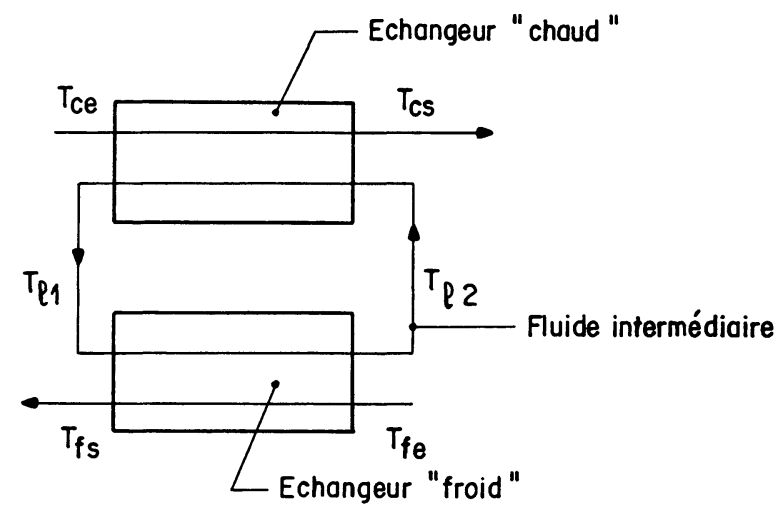

Fig. 3. - Echangeurs couplés par un fluide intermédiaire. [Fluid-coupled heat exchangers.]

les indices 1 et 2 correspondant aux deux extrémités de ce circuit. Les autres indices ont la signification précisée ci-dessus. Le diagramme des températures des fluides traversant les échangeurs est représenté sur la figure 4.

Supposons que l'on ait par exemple : $C_{\mathrm{c}}<C_{\mathrm{f}}<C_{\ell}$, d'après (3), les efficacités de l'échangeur " chaud " $E_{\mathrm{c}}$, de l'échangeur «froid» $E_{\mathrm{f}}$ et l'efficacité globale $E$ s'écrivent respectivement :

$$
\begin{gathered}
E_{\mathrm{c}}=\frac{T_{\mathrm{ce}}-T_{\mathrm{cs}}}{T_{\mathrm{ce}}-T_{\mathrm{\ell}_{2}}} \\
E_{\mathrm{f}}=\frac{T_{\mathrm{fs}}-T_{\mathrm{fe}}}{T_{\ell_{1}}-T_{\mathrm{fe}}} \\
E=\frac{T_{\mathrm{ce}}-T_{\mathrm{cs}}}{T_{\mathrm{ce}}-T_{\mathrm{fe}}} .
\end{gathered}
$$

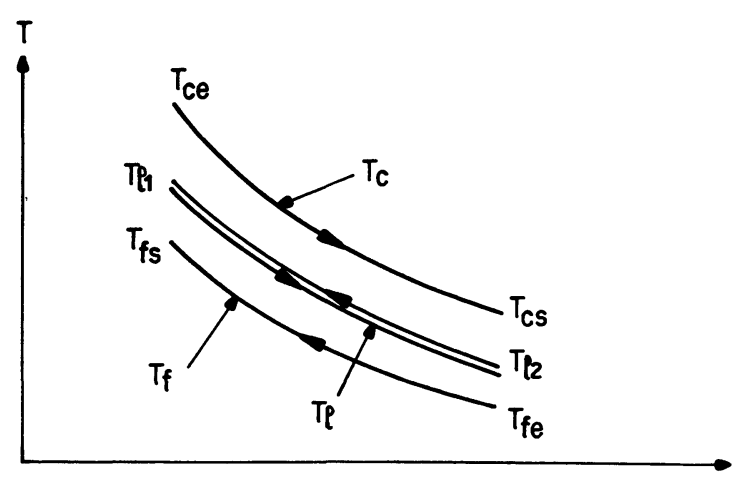

Fig. 4. - Diagramme des températures dans 2 échangeurs couplés par un fluide intermédiaire.

[Temperatures diagram in fluid-coupled heat exchangers.]
Le bilan enthalpique exprimé par la relation (1) s'écrit pour chacun des échangeurs :

$$
\begin{aligned}
C_{\mathrm{c}}\left(T_{\mathrm{ce}}-T_{\mathrm{cs}}\right) & =C_{\ell}\left(T_{\ell_{1}}-T_{\ell_{2}}\right) \\
C_{\ell}\left(T_{\ell_{1}}-T_{\ell_{2}}\right) & =C_{\mathrm{f}}\left(T_{\mathrm{fs}}-T_{\mathrm{fe}}\right) .
\end{aligned}
$$

Les relations (8) à (12) permettent d'obtenir l'expression de l'efficacité globale sous la forme :

$$
E=\frac{1}{\frac{1}{E_{\mathrm{c}}}+\frac{C_{\mathrm{c}}}{C_{\mathrm{f}}} \frac{1}{E_{\mathrm{f}}}-\frac{C_{\mathrm{c}}}{C_{\mathrm{f}}}} .
$$

Dans le cas $C_{\mathrm{f}}<C_{\mathrm{C}}<C_{\ell}$, un calcul identique aurait conduit à l'expression suivante (obtenue en permutant les indices $\mathrm{c}$ et $\mathrm{f}$ ) :

$$
E=\frac{1}{\frac{1}{E_{\mathrm{f}}}+\frac{C_{\mathrm{f}}}{C_{\mathrm{c}}} \frac{1}{E_{\mathrm{c}}}-\frac{C_{\mathrm{f}}}{C_{\ell}}} .
$$

2.4.2 Cas particulier de l'échangeur à caloducs. Pour une rangée de caloducs (transversalement aux courants de fluide externe), on pourra admettre que tous les évaporateurs sont à la même température interne, ainsi que tous les condenseurs.

On pourra également admettre que ces deux températures sont égales en négligeant la chute de température dans la phase vapeur du caloduc, ce qui est légitime.

On pourra donc considérer que la $i$-ième rangée de caloducs réalise entre les deux fluides externes un couplage analogue à la figure 3, dans lequel l'échangeur chaud est un évaporateur constitué par l'ensemble des évaporateurs des caloducs de cette rangée et l'échangeur froid, l'ensemble de leurs condenseurs avec un diagramme de température conforme à la figure 5 .

Dans ces conditions les formules générales (13) et (14) établies au § 2.4.1 s’appliquent en faisant tendre

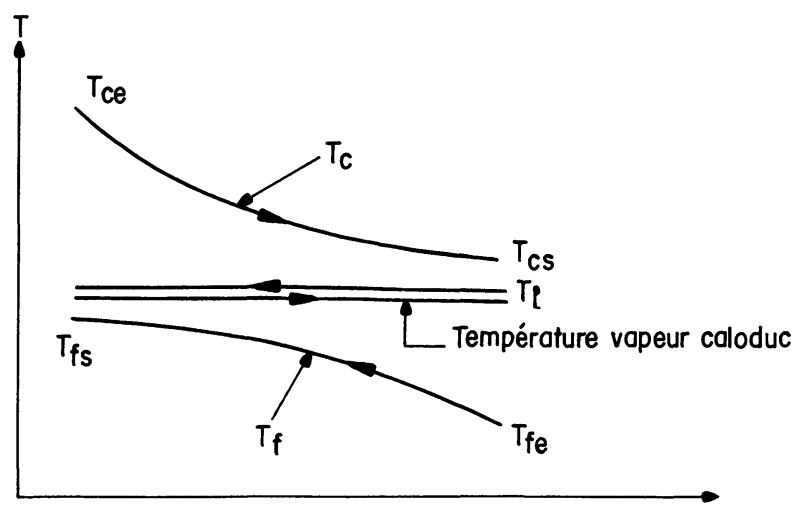

Fig. 5. - Diagramme des températures des fluides externes au passage d'une rangée de caloducs.

[Temperatures diagram of the outer fluids passing through on heat pipes row.] 
$C_{1}$ vers l'infini (ce qui correspond à $T_{\ell_{1}}=T_{\ell_{2}}$ ) et l'efficacité globale de la $i$-ième rangée de caloducs s'écrit :

$E_{i}=\frac{1}{\frac{1}{E_{\mathrm{c}, i}}+\frac{C_{\mathrm{c}}}{C_{\mathrm{f}}} \frac{1}{E_{\mathrm{f}, i}}}$

(15) pour $C_{\mathrm{c}}<C_{\mathrm{f}}$

ou

$E_{i}=\frac{1}{\frac{1}{E_{\mathrm{f}, i}}+\frac{C_{\mathrm{f}}}{C_{\mathrm{c}}} \frac{1}{E_{\mathrm{c}, i}}}$

(16) pour $C_{\mathrm{f}}<C_{\mathrm{c}}$

avec, d'après (6) et (7) :

$E_{\mathrm{c}, i}=1-\exp \left(-\frac{U_{c, i} \cdot A_{c, i}}{C_{\mathrm{c}}}\right)$
$E_{\mathrm{f}, i}=1-\exp \left(-\frac{U_{\mathrm{f}, i} \cdot A_{\mathrm{f}, i}}{C_{\mathrm{f}}}\right)$

Expression dans lesquelles, on désigne pour la $i$-ième rangée respectivement pour le côté chaud et pour le côté froid, par :

$U_{\mathrm{c}, i}$ et $U_{\mathrm{f}, i}$ les coefficients d'échange globaux entre le fluide externe et le fluide caloduc.

$A_{\mathrm{c}, i}$ et $A_{\mathrm{f}, i}$ les aires des surfaces d'échange pour l'ensemble de la rangée auxquels sont rapportés $U_{\mathrm{c}, i}$ et $U_{\mathrm{f}, i}$.

Ayant calculé l'efficacité globale de chaque rangée de caloducs, il nous reste à calculer l'efficacité globale totale $E$ des $R$ rangées de caloducs constituant l'échangeur.

Celles-ci constituent une suite d'échangeurs d'efficacité $E_{i}(i=1$ à $R)$ à contre-courant global (Fig. 6).

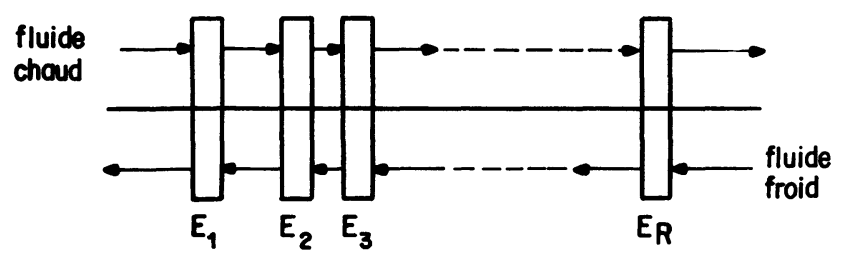

Fig. 6. - Association d'échangeurs en contre-courant global.

[Overall counter-flow heat exchangers association.]

D'après [14], on a :

$$
E=\frac{1-\prod_{i=1}^{R}\left|\frac{1-C^{*} E_{i}}{1-E_{i}}\right|}{C^{*}-\prod_{i=1}^{R}\left|\frac{1-C^{*} E_{i}}{1-E_{i}}\right|}
$$

$\operatorname{avec} C^{*}=\frac{C_{\min }}{C_{\max }}, \prod_{i=1}^{R}$ désignant le produit sur l'indice $i$ variant de 1 à $R$.

Dans le cas où toutes les rangées ont même efficacité $E_{0}$ la relation (19) devient :

RevUe de PHysique aPPLIQUée. - T. 17, No 9, SEPTEMBre 1982

$$
E=\frac{1-\left|\frac{1-C^{*} E_{0}}{1-E_{0}}\right|^{R}}{C^{*}-\left|\frac{1-C^{*} E_{0}}{1-E_{0}}\right|^{R}} .
$$

Dans le cas où $C^{*}=1$, la levée de l'indétermination sur $\left(19^{\prime}\right)$ conduit à :

$$
E=\frac{R E_{0}}{1+(R-1) E_{0}}
$$

2.5 Procédure de CAlCul. - Conformément à ce qui a été dit précédemment, la procédure de calcul consistera, à partir des propriétés thermophysiques des 2 fluides et des données géométriques de l'échangeur, à calculer d'abord les coefficients d'échange globaux rapportés à une rangée de caloducs côté chaud et froid. Ceci se fait selon les méthodes classiques en usage dans le calcul des échangeurs compacts [11] en prenant soin de tenir compte de la résistance thermique du caloduc lui-même entre paroi et vapeur que l'on aura calculé séparément.

On calculera ensuite les efficacités côté chaud et côté froid à l'aide des relations (17) et (18) puis l'efficacité globale en utilisant (15) ou (16) et (19), (19') ou $\left(19^{\prime \prime}\right)$.

On en déduira la puissance échangée et les températures de sortie des fluides à l'aide de (5) et (1). On vérifiera que le flux traversant chaque caloduc est inférieur à son flux limite.

Si les résultats obtenus conviennent sur le plan thermique on pourra calculer les pertes de charge côté chaud et côté froid toujours selon les méthodes de calcul des échangeurs compacts [11].

L'organigramme ci-après, donné à titre indicatif, résume la procédure que nous venons de décrire.

3. Etude de quelques paramètres particuliers aux échangeurs à caloducs. - 3.1 GÉNÉRALITÉs. - Nous n'examinerons pas ici l'influence des paramètres communs à tous les échangeurs compacts, ils concernent pour la plupart la géométrie de la surface d'échange, que l'on cherche à optimiser afin d'obtenir le meilleur coefficient d'échange avec le minimum de pertes de charge.

Signalons simplement que les surfaces couramment utilisées pour les échangeurs à caloducs sont :

- Les tubes ailetés (ailettes circulaires en hélice, ailettes carrées, ailettes segmentées).

- Les faisceaux de tubes avec ailettes sous forme de plaques ininterrompues communes à tout le faisceau.

Ce dernier type de surface est souvent utilisé dans le domaine des basses températures (climatisation) à cause de sa compacité. Les tubes ailetés sont en général préférés dans le domaine des moyennes températures $\left(250\right.$ à $\left.500^{\circ} \mathrm{C}\right)$ à cause de la meilleure tenue en tempé- 
Calcul des propriétés

thermophysiques des fluides

Calcul des débits de capacité des fluides

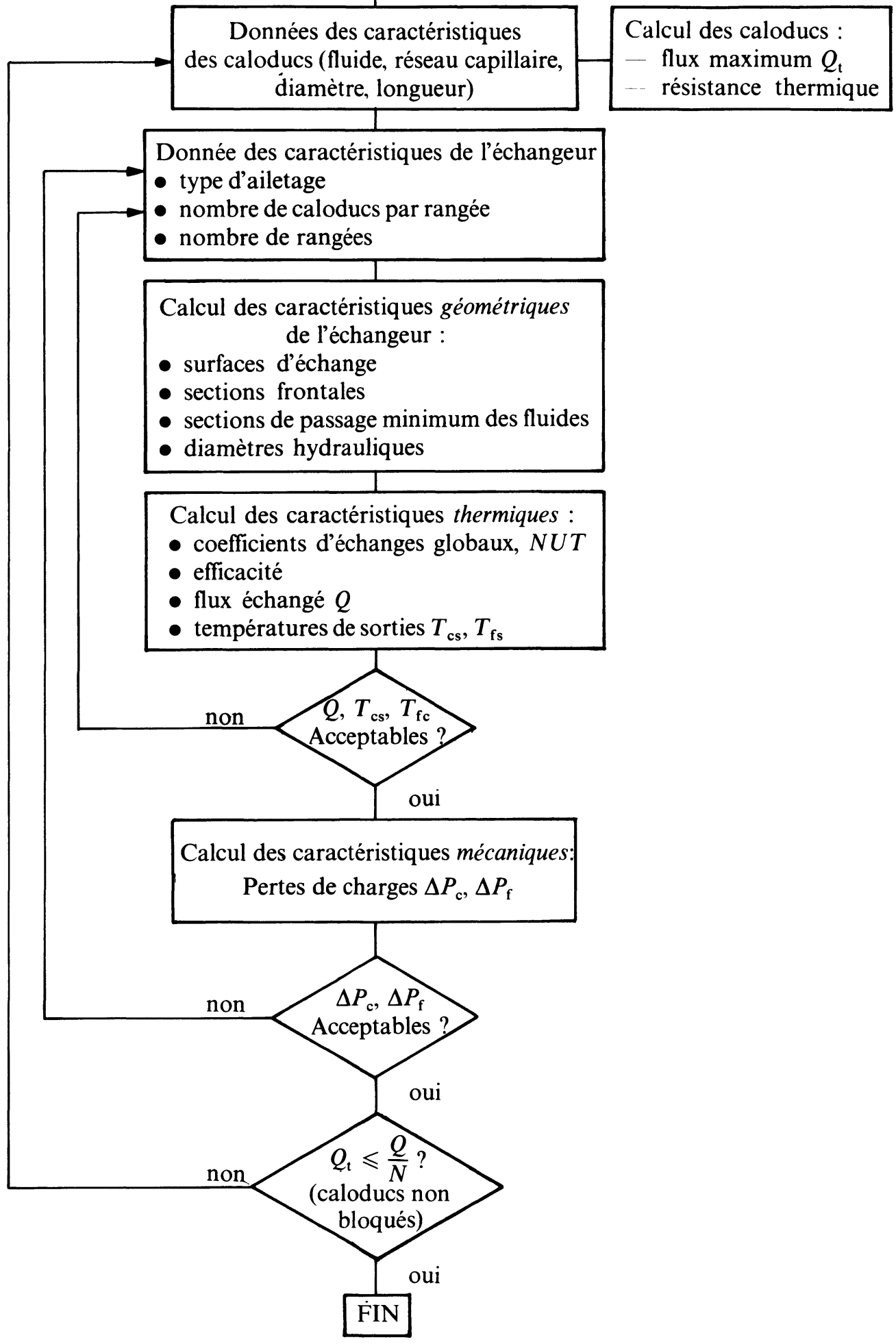


rature de certains types d'ailettes. On notera que dans le cas des tubes acier ce sont les ailettes segmentées qui donnent les meilleurs résultats [12].

Le nombre des caloducs est déterminé à partir de la surface d'échange externe nécessaire et du flux maximum à échanger compte tenu du flux limite transportable par le type de caloduc choisi, à la température de fonctionnement. Il peut varier de quelques dizaines à plusieurs centaines. Les diamètres de tubes couramment employés vont de 12 à $30 \mathrm{~mm}$, sachant que le flux limite est grossièrement proportionnel à la section. Les flux usuels par caloduc vont de 1 à $4 \mathrm{~kW}$, la puissance de l'échangeur pouvant aller de quelques dizaines à un millier de $\mathrm{kW}$.

Nous étudions ci-dessous l'influence de quelques paramètres propres aux échangeurs à caloducs, à savoir le type de structure capillaire, l'inclinaison et les conditions démarrage.

3.2 StRUCTURE CAPILLAIRE INTERNE DES CALODUCS. La structure capillaire interne d'un caloduc agit par le phénomène de pompage capillaire. Elle a un double rôle :

1) Assurer le retour du condensat à l'évaporateur dans le cas où l'effet de la gravité s'y oppose ou est insuffisant (caloducs horizontaux par exemple).

2) Homogénéiser le film liquide sur toute la surface de l'évaporateur de manière à assurer une évaporation sans ébullition avec une faible surchauffe et sans " points chauds".

Le premier rôle est le moteur du fonctionnement du caloduc lorsqu'il n'y a pas assistance de la gravité mais il perd de son importance dans le cas où l'évaporateur est plus bas que le condenseur.

Le second rôle est une amélioration des performances du caloduc par diminution de sa résistance thermique interne mais il n'est pas absolument indispensable.

Ainsi on peut envisager des caloducs placés verticalement évaporateur en bas sans structure capillaire interne. Ces éléments appelés thermosyphons présentent l'avantage d'un faible prix de revient (ce sont de simples tubes scellés) et l'inconvénient d'un écart de température plus grand entre les extrémités (résistance thermique supérieure) ainsi qu'un certain risque d'instabilité de fonctionnement.

Diverses structures capillaires sont présentées sur la figure 7, le choix résultera d'un compromis entre les performances, le prix de revient et les possibilités de fabrication. Notons que les structures rainurées semblent être une assez bonne solution pour les caloducs utilisés dans les échangeurs.

3. 3 InCLINAison. - La gravité joue un rôle déterminant dans le fonctionnement du caloduc en favorisant ou en contrariant le retour du condensat vers l'évaporateur, il en résulte que l'inclinaison du caloduc déterminera son mode de fonctionnement.
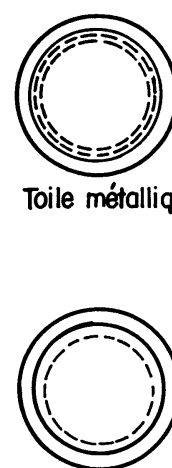

Toile métallique avec espace onnulair
Toile métallique

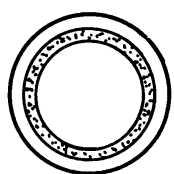

Poudre métallique frittée
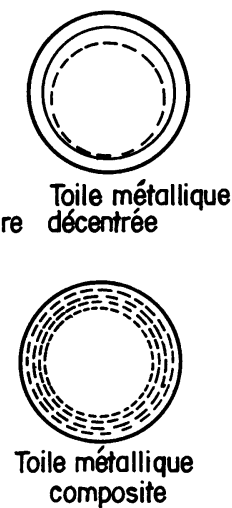
composite

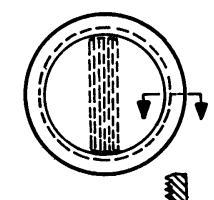

Rainures circulaires avec mèche axiale
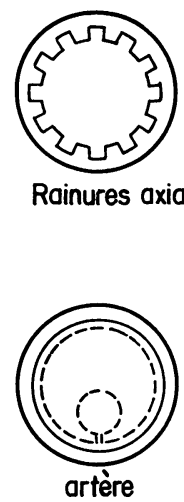

Rainures axiales

artere

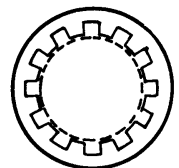

Rainures axiales ovec toile écran

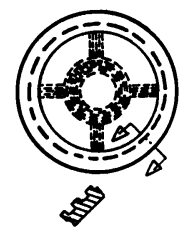

Rainures circulaires ovec tunnel
Fig. 7. - Exemples de structures capillaires.

[Examples of wick structures.]

La limite du flux transportable par un caloduc, due au débit de retour du condensat, a l'allure indiquée sur la figure 8 . On voit qu'il existe une valeur optimum d'inclinaison pour obtenir le flux limite maximum. Cette inclinaison optimum dépend des caractéristiques

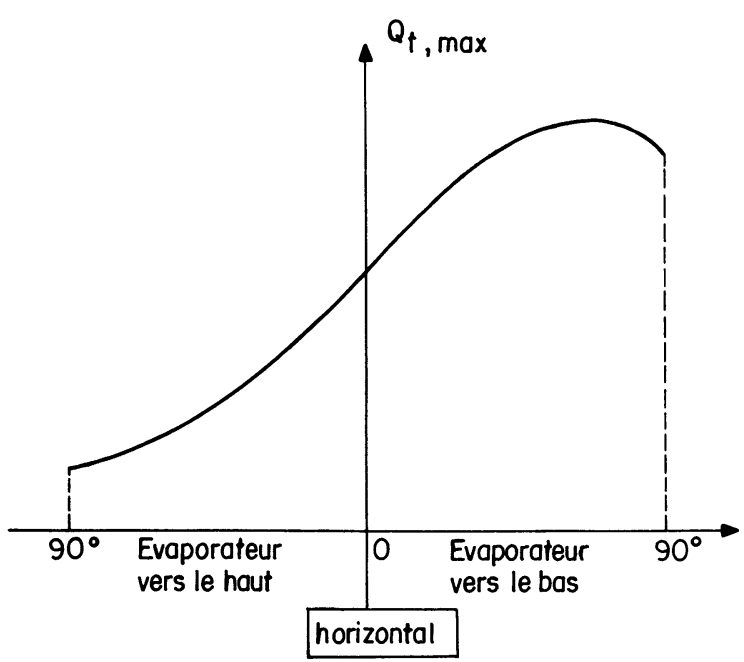

Fig. 8. - Flux thermique maximum dans un caloduc en fonction de l'angle d'inclinaison.

[Maximum heat flux versus tilt for a heat pipe.] 
du réseau capillaire choisi, et peut être la verticale (évaporateur en bas).

Dans le cas où l'on recherche la puissance transmise maximum on placera les caloducs dans l'échangeur de manière à ce qu'ils travaillent sous leur inclinaison optimum.

Dans le cas où l'on désire pouvoir transmettre la chaleur dans l'un ou l'autre sens selon les températures relatives des deux veines fluides, on placera les caloducs horizontaux (c'est le cas par exemple dans les installations de conditionnement d'air où l'on peut avoir besoin selon la saison de réchauffer ou de refroidir l'air entrant à partir de l'air sortant).

Enfin, dans le cas où l'on désire moduler la puissance transférée ou dégivrer l'échangeur on peut utiliser un dispositif à inclinaison variable (breveté) [13].

3.4 DémarRage. - Le problème du démarrage $\mathrm{du}$ fonctionnement des caloducs se pose durant la phase de montée en température de l'échangeur car, lorsqu'un caloduc se trouve très en dessous de sa température nominale de fonctionnement, la pression de vapeur saturante qui règne à l'intérieur est très faible et diminue grandement le flux limite transportable (limite sonique). Il faut par conséquent veiller à ce que, durant toute la phase de démarrage, le flux thermique "appelé " à travers l'échangeur n'excède pas la limite sonique des caloducs.

En fait, ce problème ne se pose pas pour les caloducs à basse température $\left(20-150^{\circ} \mathrm{C}\right)$ où la pression de vapeur saturante de fluides tels que l'eau, les alcools, les fréons, l'ammoniaque ou l'acétone est suffisante à la température ambiante.

Pour les caloducs à moyenne température utilisant le Gilotherm DO, le Dowtherm A (150-300 $\left.{ }^{\circ} \mathrm{C}\right)$ ou le mercure $\left(200-500^{\circ} \mathrm{C}\right)$ la question doit être examinée attentivement et peut nécessiter une suppression de la convection forcée (soufflage) côté froid durant la montée en température (ce point fait partie d'une étude expérimentale actuellement en cours).

Enfin dans le domaine des hautes températures $\left(500-1000^{\circ} \mathrm{C}\right)$ avec les fluides tels que le potassium ou le sodium, qui sont solides à température ambiante, la question devient critique. Il peut alors être nécessaire d'envisager, dans la phase transitoire, un préchauffage du fluide froid de manière à maintenir un faible écart de température entre les deux veines fluides tant que la température de fonctionnement n'est pas atteinte.

Précisons que dans tous les cas, lorsque les précautions que nous venons d'indiquer sont prises, le principe même du caloduc conduit à un temps de réponse très faible de l'échangeur, d'où une excellente efficacité de la récupération de chaleur dans les régimes transitoires.

4. Conclusion. - Les échangeurs à caloducs, déjà utilisés dans le domaine de la climatisation, sont susceptibles d'une large utilisation dans le domaine industriel pour la récupération de chaleur à moyenne et haute température.

Leur principe se prête bien à une construction modulaire et donne une grande souplesse dans le dimensionnement. L'utilisation d'un grand nombre d'éléments identiques assure, outre les avantages de l'effet de série, une excellente fiabilité de l'appareil (la défaillance de quelques caloducs ayant très peu d'influence sur les performances de l'échangeur entier).

Ce type d'échangeur est donc susceptible de prendre une place de choix dans la lutte pour les économies d'énergie en milieu industriel sous réserve que les problèmes technologiques qui subsistent dans le domaine des hautes températures (résistance à la corrosion notamment) soient complètement résolus.

\section{Bibliographie}

[1] Dunn, P. D., Reay, D. A., Heat Pipes (Pergamon Press) 1976.

[2] CHI, S. W., Heat Pipe. Theory and practice (McGrawHill) 1976.

[3] Ruch, M. A., Heat Pipe thermal recovery units. Conférence IECEC 1975.

[4] Reay, D. A., Heat Pipe exchangers : A review of its status and its potential in process heat recovery. Energy for industry Symposium 1978, Londres (Ed. Pergamon Press) pp. 239-256.

[5] RuCh, M. A., Grover, G. M., Heat pipe recovery unit applications 2nd International Heat Pipe Conference. Bologne (1976).

[6] Keddy, E. S., Ranken, W. A., Ceramic heat Pipes for furnace heat recovery (AIChE CEP Dec.) 1979, pp. 35-37.

[7] Reay, D. A., Searle, N. K., Forster, E. L., Comparative Study of rotaring Regenerators and Heat Pipe Exchangers.
[8] Amode, J. O., Analysis of a heat-pipe heat exchanger. $\mathrm{Ph}$. D. Univ. of New xexico. Albuquerque (1976).

[9] Feldman, K. T., Lu, D. C., Preliminary design study of heat pipe heat exchangers. 2nd International heat pipe Conference. Bologne (1976).

[10] SAC̣Adura, J. F., Initiation aux Transferts Thermiques (Technique et Documentation) 1980.

[11] Kays, W. M., London, A. L., Compact Heat Exchangers (McGraw-Hill) 1964.

[12] LU, D. C., Feldman, K. T., Cost effectiveness study of heat pipe heat exchangers. ASME Winter annual meeting (Nov. 1977).

[13] Sté Hugues Aircraft Company. Echangeur de chaleur à tubes à chaleur. Institut National de la Propriété Industrielle. Demande de Brevet no 7803973 du 13/02/78.

[14] Domingos, J. D., Analysis of complex assemblies of Heat Exchangers. Int. J. Heat Mass Transfer 12 (1969) 537-548. 